\title{
Seismic Attributes from Ultra-Thin Layered Reservoir
}

\author{
A. Stovas' ${ }^{1}$, M. Landro' and N. Janbu ${ }^{2}$ \\ 1 Department of Petroleum Engineering and Applied Geophysics, Norwegian University of Science and Technology (NTNU), \\ S.P.Andersensvei 15A, NO-7491 Trondheim - Norway \\ 2 Statoil Forskningssenter, Arkitekt Ebbells vei 10, Rotvoll, 7005 Trondheim - Norway \\ e-mail: alexey@ipt.ntnu.no
}

\begin{abstract}
Résumé - Attributs sismiques d'un réservoir à couches ultra fines - Nous proposons d'utiliser la méthode d'attributs AVO sismiques (ordonnée à l'origine et pente) à partir d'un modèle géologique ultra fin basé sur le logiciel de modélisation SBED. Le logiciel SBED repose sur la manipulation de fonctions sinusoïdales qui créent des surfaces représentant une sédimentation progressive. Le déplacement des surfaces génère une image en trois dimensions réaliste en termes de sédimentologie et permettant de reconstituer avec précision des environnements de dépôt aussi divers que des chenaux de marée et des flux de masse. Au volume du dépôt modélisé, on peut associer des informations pétrophysiques, créant des propriétés intrinsèques telles que la porosité et la perméabilité (verticales et horizontales). La technique de calcul de la moyenne de Backus est utilisée pour la mise à l'échelle centimétrique (le rapport volumique sable-argile contrôle les propriétés acoustiques des couches ultra fines). Il en résulte des données pseudo-logs comprenant les paramètres anisotropiques intrinsèques. La modélisation sismique utilise la méthode de propagation de matrice et permet de prendre en compte tous les multiples en mode pur. Les attributs AVO qui en résultent seront dépendants de la fréquence. Dans le cadre de ce modèle ultra fin, nous pouvons tester différents scénarios de saturation de fluides et quantifier la probabilité d'éventuels analogues composites. Cette modélisation peut également être utilisée pour inverser des données sismiques réelles et retrouver le rapport sable-argile et la saturation des fluides pour les réservoirs ultra fins.
\end{abstract}

\begin{abstract}
Seismic Attributes from Ultra-Thin Layered Reservoir - We propose the method of computation seismic AVO attributes (intercept and gradient) from ultra-thin geological model based on the SBED modelling software. The SBED software is based on manipulating sine-functions, creating surfaces representing incremental sedimentation. Displacement of the surfaces creates a three dimensional image mimicking bedform migration, and depositional environments as diverse as tidal channels and mass flows can be accurately recreated. The resulting modelled deposit volume may be populated with petrophysical information, creating intrinsic properties such as porosity and permeability (both vertical and horizontal). The Backus averaging technique is used for up-scaling within the centimetre scale (the intrinsic net-to-gross value controls the acoustic properties of the ultra-thin layers). It results in pseudolog data including the intrinsic anisotropy parameters. The synthetic seismic modelling is given by the matrix propagator method allows us to take into account all pure mode multiples, and resulting AVO attributes become frequency dependent. Within this ultra-thin model we can test different fluid saturation scenarios and quantify the likelihood of possible composite analogues. This modelling can also be used for inversion of real seismic data into net-to-gross and fluid saturation for ultra-thin reservoirs.
\end{abstract}




\section{INTRODUCTION}

Hydrocarbon reservoir characterization is the process of creating an interdisciplinary understanding of reservoir complexity to accurately evaluate recoverable volumes. For most modern hydrocarbon reservoirs, 3D seismic is commonly used to characterize depositional geometries and fluid saturations and to generate the initial reservoir geo-model. Well data that is added after a discovery represents isolated sampling points which laterally only hold few $\mathrm{cm}$ of geological information.

Seismic resolution is normally characterized by a voxel size about $15-25 \mathrm{~m}$ (both horizontal and vertical). From the 3D seismic data a common situation is that top and base reservoir are interpretable, while the internal boundaries in the reservoir need to be located based on well bore information. These intrareservoir boundaries are then interpolated between well locations. Geological software packages today give very detailed geological models, but these still have significant uncertainty at the subseismic scale (Avseth et al., 2005).

The results presented in this paper come from the integration of sedimentary deposit modelling as well as seismic modelling (Wen, 2004; Nordahl et al., 2005; Stovas et al., 2006; Stovas and Arntsen, 2006). The proposed methodology can be used for the modelling of seismic in order to test the realistic sedimentology and fluid saturation scenario.

\section{INTERBEDDED MULTIPLES}

The most realistic seismic model reproducing the sedimentary basin is the stack of thin layers. Typical sonic logs show that the sedimentary rocks are composed of layers with thicknesses of order of $0.1 \mathrm{~m}$ to $10 \mathrm{~m}$. Sometimes a micro layering of even smaller characteristic sizes can be observed (Shapiro and Hubral, 1999).

Multiple reflected and transmitted seismic waves are usually neglected in standard seismic modeling technology. This assumption results in very simplistic convolutional seismic model which takes into account the primary reflections only. Although this assumption is valid for the thick layers model only (with separated reflection events), it has been automatically extended for a finely layered medium. For multilayered model with very thin layers, the interbedded multiples play extremely important role interfering with primary reflections.

If we consider the influence of thinly layered structures on transmitted seismic wavefields only, this effect is called stratigraphic filtering (Shapiro and Hubral, 1999; Stovas and Arntsen, 2006). On another hand, the thinly layered structure is also important for reflected seismic wavefield, as it was shown for periodic medium by Stovas and Ursin (2005). Stovas et al. (2006) developed a method to invert the AVO attributes estimated from the top of thin sand-shale interbedded
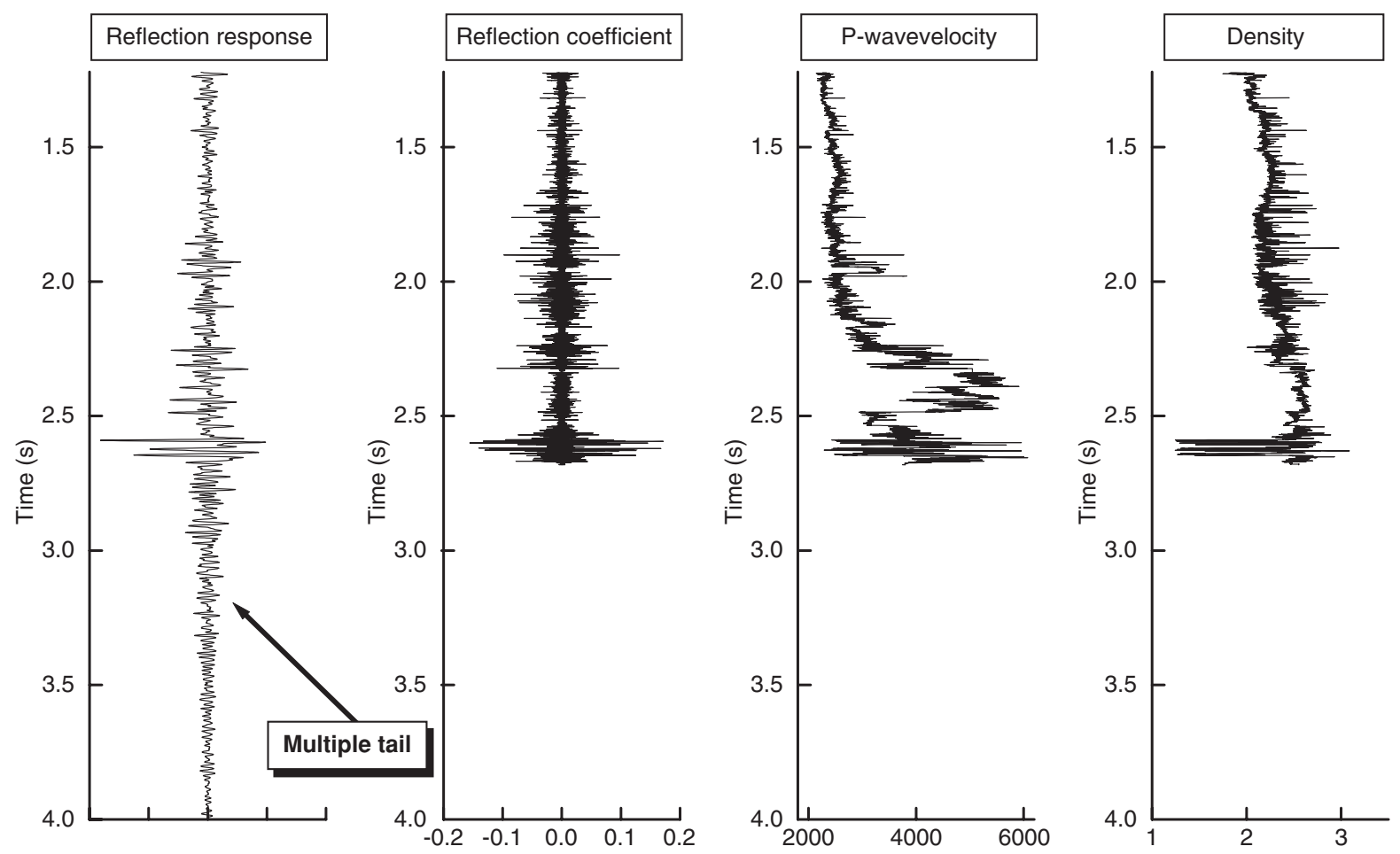

Figure 1

P-wave velocity and density profiles shown in two-way vertical traveltime scale (two columns to the right), the reflection coefficient time series and the reflection response (column to the left). 
reservoir into net-to-gross and water saturation. They shown how net-to-gross controls the reflection attributes and discussed the heterogeneity versus periodicity effects.

The standard procedure to compute synthetic seismic response from the well-log data includes:

- blocking of elastic properties based on the geological interpretation of the well-log data;

- convolution of the reflection coefficient time series with seismic wavelet.

In this flow the effect of interbedded multiples is neglected. While, in thin-layered models this effect results in velocity dispersion as well as interbedded resonances due to multiples contribution.

This effect is illustrated by using well-log data from the North Sea (Fig. 1). The velocity and density profiles, shown to the right, result in quite strong contrast or reflection coefficient time series. The synthetic seismic response computed from the full wave modeling is shown to the right and has significant amplitudes at the times below the base of the model. This is so called multiples tail. This tail is result of all interbedded multiples, and its contribution is strongly affected by the contrast in elastic properties and the dominant wavelength to spatial sampling rate (Stovas and Arntsen, 2006). In Figure 2 we compare the seismic response computed from the full wave modeling, simple convolution from well-log data and simple convolution from the detailed blocked well-log data. One can see that the responses are different both in amplitude and phase.

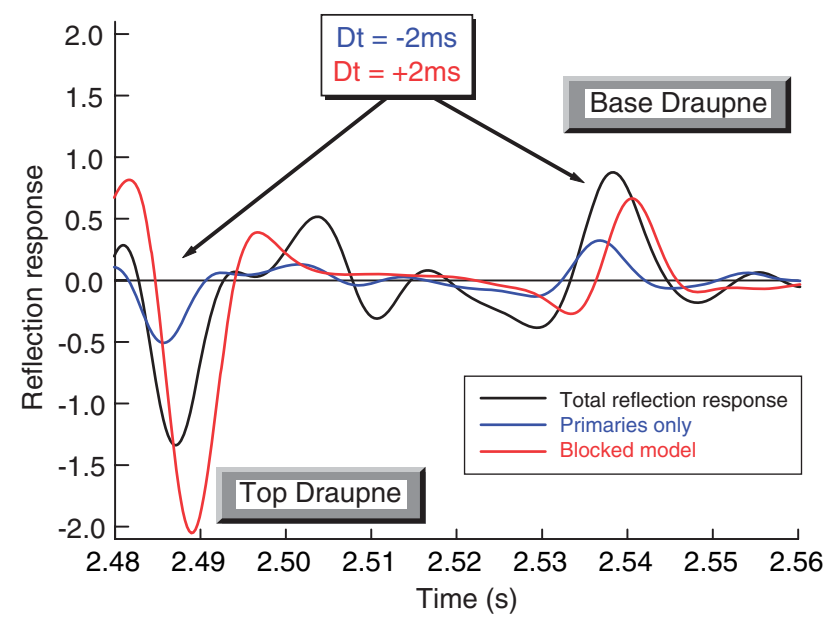

Figure 2

Reflection response computed from well-log data shown in Figure 1 with full wave modelling (black line) and convolutional modelling (blue line) and from detailed blocked well$\log$ data with convolutional modelling (red line). The top and base reservoir are indicated. The time-shift between the positions for both top and base reservoir from different modelling techniques varies between $-2 \mathrm{~ms}$ to $+2 \mathrm{~ms}$.

\section{METHODOLOGY}

The methodology consists of two very important parts: SBED modeling introducing the sedimentology scenario and seismic reflection modeling based on the matrix propagator method.

\subsection{SBED Model}

The SBED software is based on manipulating sine-functions, creating surfaces representing incremental sedimentation (Wen, 2004; Nordahl et al., 2005). Displacement of the surfaces creates a three-dimensional image mimicking bedform migration, and depositional environments as diverse as tidal channels and mass flows can be accurately recreated. Due to the high-resolution output, common practice is to generate models that are volumetrically slightly larger than real core data (30 $\times 30 \mathrm{~cm}$ in $x$ and $y$ directions). The resulting modelled deposit volume may be populated with petrophysical information, creating intrinsic properties such as porosity and permeability (both vertical and horizontal). These petrophysical properties are based on empirical Gaussian distributions that can be further customized to fit observed data. In addition, a detailed net-to-gross ratio is produced for each modelled case.

\subsection{Synthetic Modelling}

From the SBED model we obtain the net-to-gross and porosity logs defined for $1 \mathrm{~cm}$ scale. With help the Gassmann (1951) model and porosity log, we introduce the fluid saturation scenario and define the elastic parameters for sand. Then we apply the binary Backus averaging (Backus, 1962) using net-to-gross log and the typical values for elastic parameters for shale and obtain the effective elastic parameters (including the Thomsen parameters for the layer induced VTI anisotropy) at $1 \mathrm{~cm}$ scale. The typical elastic properties for sand and shale can be obtained from laboratory measurements.

The approximate equation for the anisotropy parameters is given by (Stovas et al., 2006).

$$
\begin{aligned}
& \varepsilon=2 \gamma_{1}^{2}(N / G)(1-(N / G)) \Delta c_{44}\left(\Delta c_{33}-\gamma_{1}^{2} \Delta c_{44}\right) \\
& \delta=(N / G) \gamma_{1}^{2}\left(1-2 \gamma_{1}^{2}(N / G)\right) \Delta c_{44}\left(\Delta c_{33}-\Delta c_{44}\right)
\end{aligned}
$$

where $\gamma_{i}$ is $V s / V p$ ratio for shale, $(N / G)$ is the net-to-gross ratio and $\Delta c_{33}$ are $\Delta c_{44}$ the contrasts in the corresponding values for the stiffness coefficients between shale and sand.

To compute the AVO attributes from the layered reservoir we use the matrix propagator method (Kennett, 1983) which results in analytic expressions for periodically layered medium (Stovas and Ursin, 2005). 
The propagator matrix for one cycle:

$$
\begin{aligned}
S=\frac{1}{1-r^{2}}\left(\begin{array}{cc}
e^{i \theta_{1}} & 0 \\
0 & e^{-i \theta_{1}}
\end{array}\right)\left(\begin{array}{cc}
1 & r \\
r & 1
\end{array}\right) \\
\left(\begin{array}{cc}
e^{i \theta_{2}} & 0 \\
0 & e^{-i \theta_{2}}
\end{array}\right)\left(\begin{array}{cc}
1 & -r \\
-r & 1
\end{array}\right)=\left(\begin{array}{cc}
a & b \\
b^{*} & a^{*}
\end{array}\right)
\end{aligned}
$$

with velocities $v_{1}$ and $v_{2}$, densities $\rho_{1}$ and $\rho_{2}$ and the thicknesses $d_{1}$ and $d_{2}$. For a given frequency $f$ the phase factors are: $\theta_{k}=2 \pi f d_{k} / v_{k}=2 \pi f \Delta t_{k}$, where $\Delta t_{k}$ is the traveltime in medium $k$ for one cycle. The normal incidence reflection coefficient at the interface between the layers is given by:

$$
r=\frac{\rho_{2} v_{2}-\rho_{1} v_{1}}{\rho_{2} v_{2}+\rho_{1} v_{1}}
$$

The propagator matrix (2) can be represented by the eigenvalue decomposition:

$$
S=U \Sigma U^{-1}
$$

where $\Sigma=\operatorname{diag}\left[\sigma_{1}, \sigma_{2}\right]$ with:

$$
\sigma_{1,2}= \begin{cases}\operatorname{Re} a \pm i \sqrt{1-(\operatorname{Re} a)^{2}} & \text { for }|\operatorname{Re} a|<1 \\ \operatorname{Re} a \mp \sqrt{(\operatorname{Re} a)^{2}-1} & \text { for }|\operatorname{Re} a| \geq 1\end{cases}
$$

and the matrix:

$$
U=\left(\begin{array}{cc}
1 & 1 \\
\left(\sigma_{1}-a\right) / b & \left(\sigma_{2}-a\right) / b
\end{array}\right)
$$

A stack of $M$ cycles of total thickness $D=M d=M\left(d_{1}+d_{2}\right)$ has the propagator matrix:

$$
\begin{aligned}
& Q=S^{M}=U \sum^{M} U^{-1}=\frac{1}{u_{22}-u_{21}} \\
& \left(\begin{array}{cc}
\sigma_{1}^{M} u_{22}-\sigma_{2}^{M} u_{21} & \sigma_{2}^{M}-\sigma_{1}^{M} \\
-u_{21} u_{22}\left(\sigma_{2}^{M}-\sigma_{1}^{M}\right) & \sigma_{2}^{M} u_{22}-\sigma_{1}^{M} u_{21}
\end{array}\right)
\end{aligned}
$$

with $u_{21}=\left(\sigma_{1}-a\right) / b$ and $u_{22}=\left(\sigma_{2}-a\right) / b$. Another way to compute the propagator or transfer matrix is to exploit the Cailey-Hamilton theorem to establish relation between $S^{2}$ and $S$ which results in the recursive relation for Chebychev polynomials.

The transmission and reflection responses for a downgoing wave at the top of the layers are:

$$
\begin{aligned}
& t_{D}=q_{22}^{-1}=\frac{\sigma_{2}-\sigma_{1}}{\left(\sigma_{2}-a\right) \sigma_{2}^{M}-\left(\sigma_{1}-a\right) \sigma_{1}^{M}} \\
& r_{D}=q_{12} q_{22}^{-1}=\frac{\left(\sigma_{2}^{M}-\sigma_{1}^{M}\right) b}{\left(\sigma_{2}-a\right) \sigma_{2}^{M}-\left(\sigma_{1}-a\right) \sigma_{1}^{M}}
\end{aligned}
$$

with $q_{i j}, i, j=1,2$ being the elements of propagator matrix $Q$ given in (7). After algebraic manipulations, Equation (8) can be written as:

$$
\begin{gathered}
t_{D}=\frac{\sin \varphi}{\sin \varphi \cos M \varphi-i \operatorname{Im} a \sin M \varphi} \\
=\frac{\cos M \varphi+i \sin M \varphi \frac{\operatorname{Im} a}{\sin \varphi}}{1+\sin ^{2} M \varphi\left[\left(\frac{\operatorname{Im} a}{\sin \varphi}\right)^{2}-1\right]}=\frac{e^{i \alpha}}{\sqrt{1+C^{2}}} \\
r_{D}=\frac{b \sin M \varphi}{\sin \varphi \cos M \varphi-i \operatorname{Im} a \sin M \varphi} \\
=t_{D} b \frac{\sin M \varphi}{\sin \varphi}=\frac{\left.C e^{i\left(\alpha+\theta_{1}+\frac{\pi}{2}\right.}\right)}{\sqrt{1+C^{2}}}
\end{gathered}
$$

where $\varphi$ is the phase of the eigenvalues (5), $\alpha$ and $C$ being the phase and amplitude factors, respectively.

These equations represent the total transmission and reflection responses from the stack of $M$ cycles.

\subsection{AVO Attributes}

The AVO attributes can be represented by two terms Taylor series with respect to sine of the incident angle. Expanding the reflection response equation from (Stovas and Ursin, 2005) results in:

$$
R=R(0)+G \sin ^{2} \theta
$$

where $R(0)$ and $G$ are intercept and gradient, respectively. Note that for a finely layered medium both AVO attributes are frequency dependent functions. Multiplying them with the

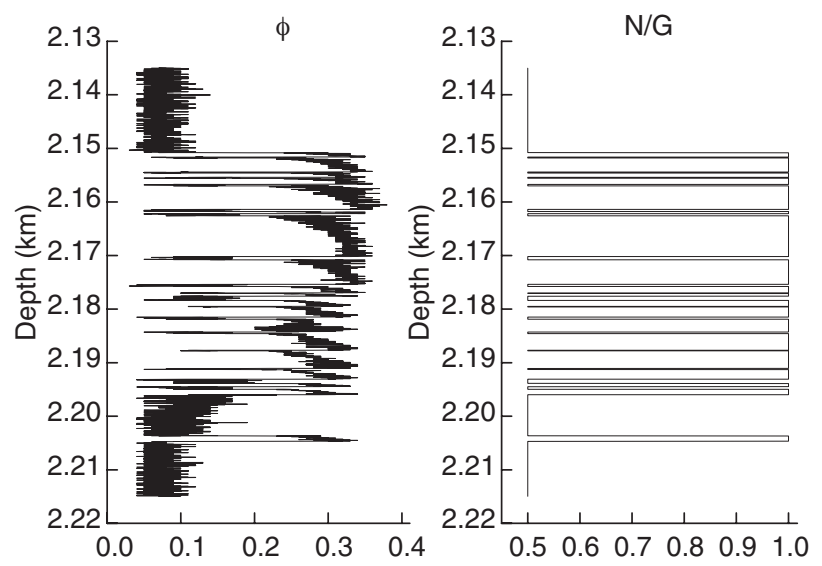

Figure 3

The porosity (to the left) and net-to-gross (to the right) vertical profiles generated by SBED for the reservoir zone. 

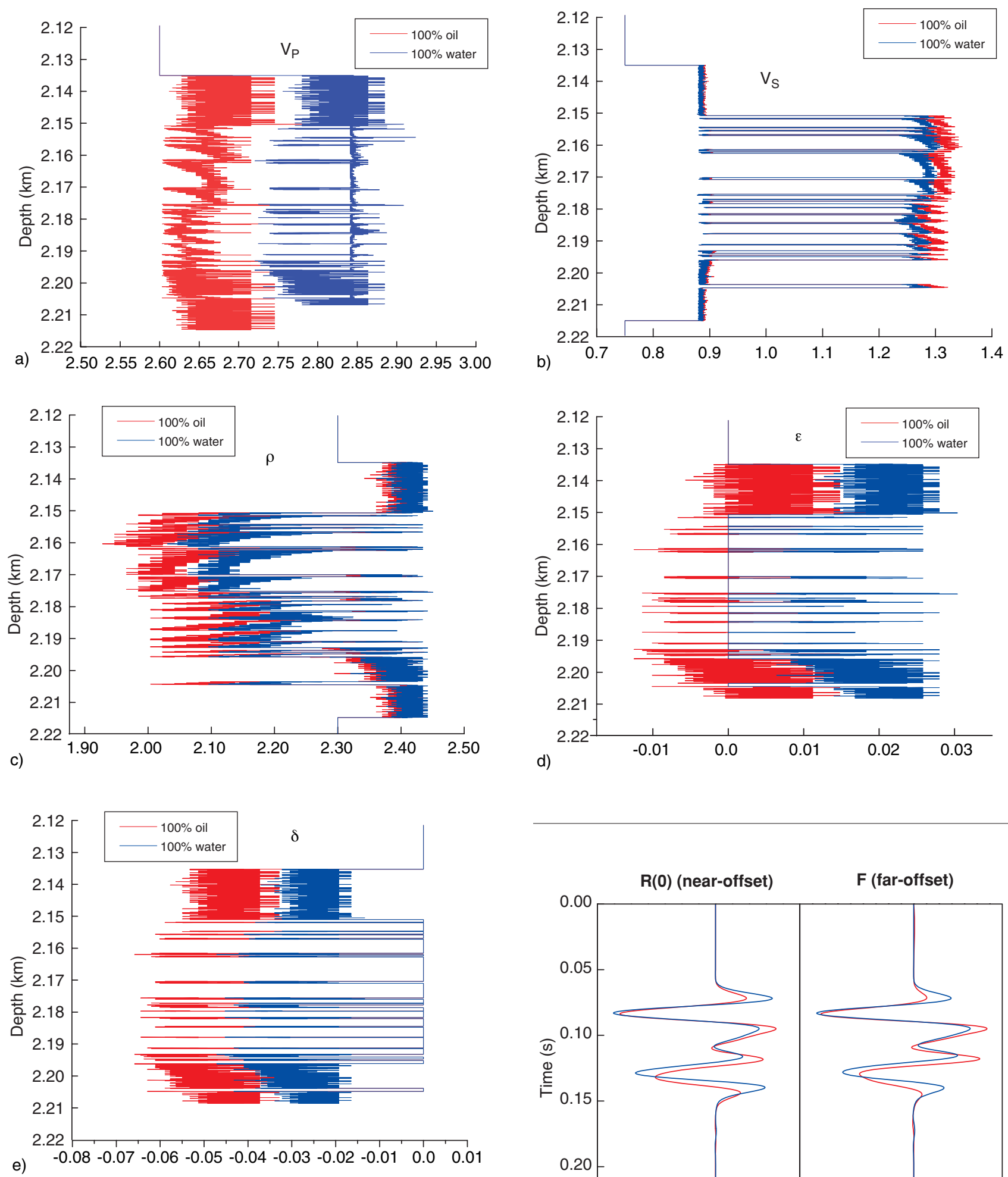

Figure 4

The elastic properties: P-wave velocity (a), S-wave velocity (b), density (c), anisotropic parameters epsilon (d) and delta (e) for two fluid scenarios (water saturation case is shown by blue line and oil saturation case is shown by red line). The properties were computed from porosity and net-to-gross shown in Figure 3 and typical rock physics parameters.

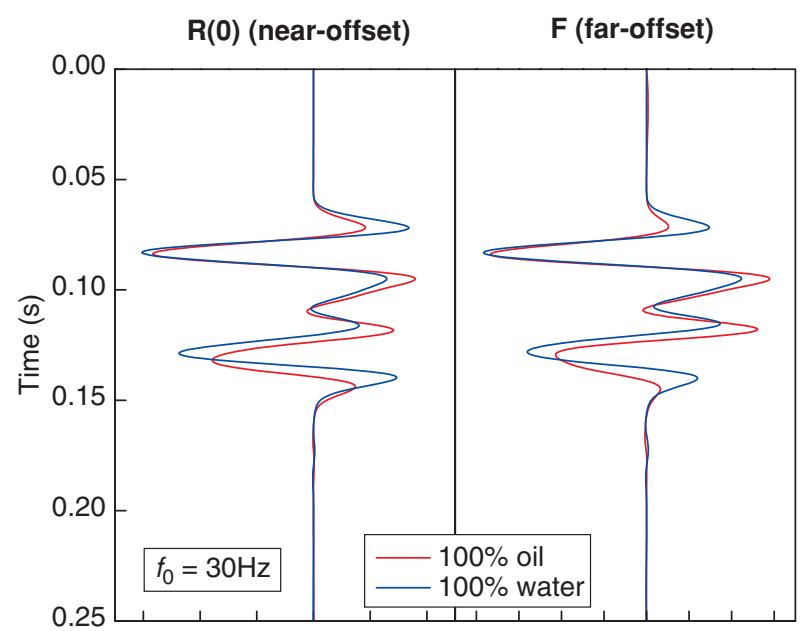

Figure 5

The near and far-offset attributes computed with full wave modelling from the models shown in Figure 4. The central frequency of the wavelet is $20 \mathrm{~Hz}$ (top) and $30 \mathrm{~Hz}$ (bottom). 

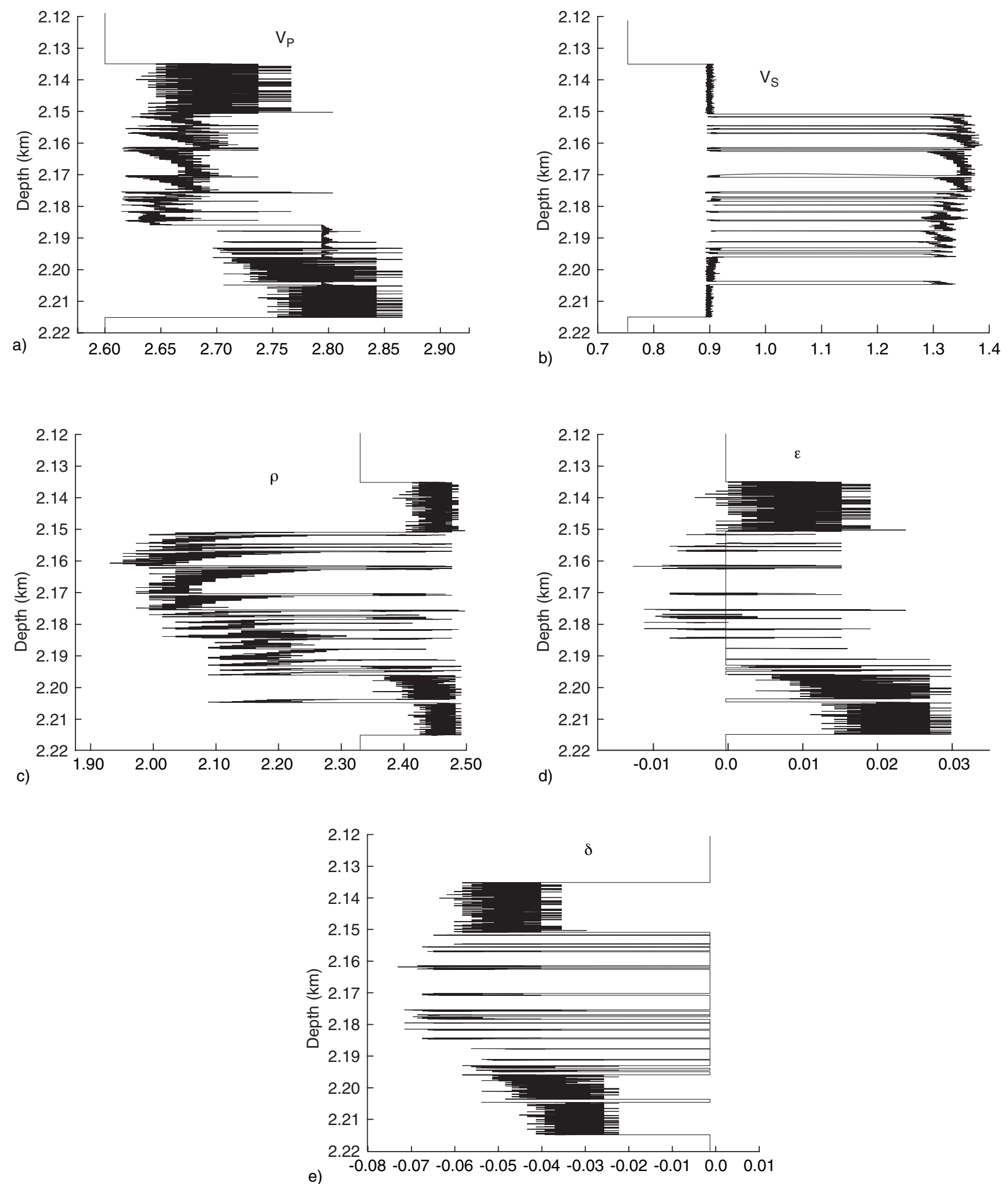

Figure 6

The elastic properties: P-wave velocity (a), S-wave velocity (b), density (c), anisotropic parameters epsilon (d) and delta (e) for oil-water contact scenario ( $20 \%$ water saturation above and $80 \%$ water saturation below the contact). The properties were computed from porosity and net-to-gross shown in Figure 3 and typical rock physics parameters. 

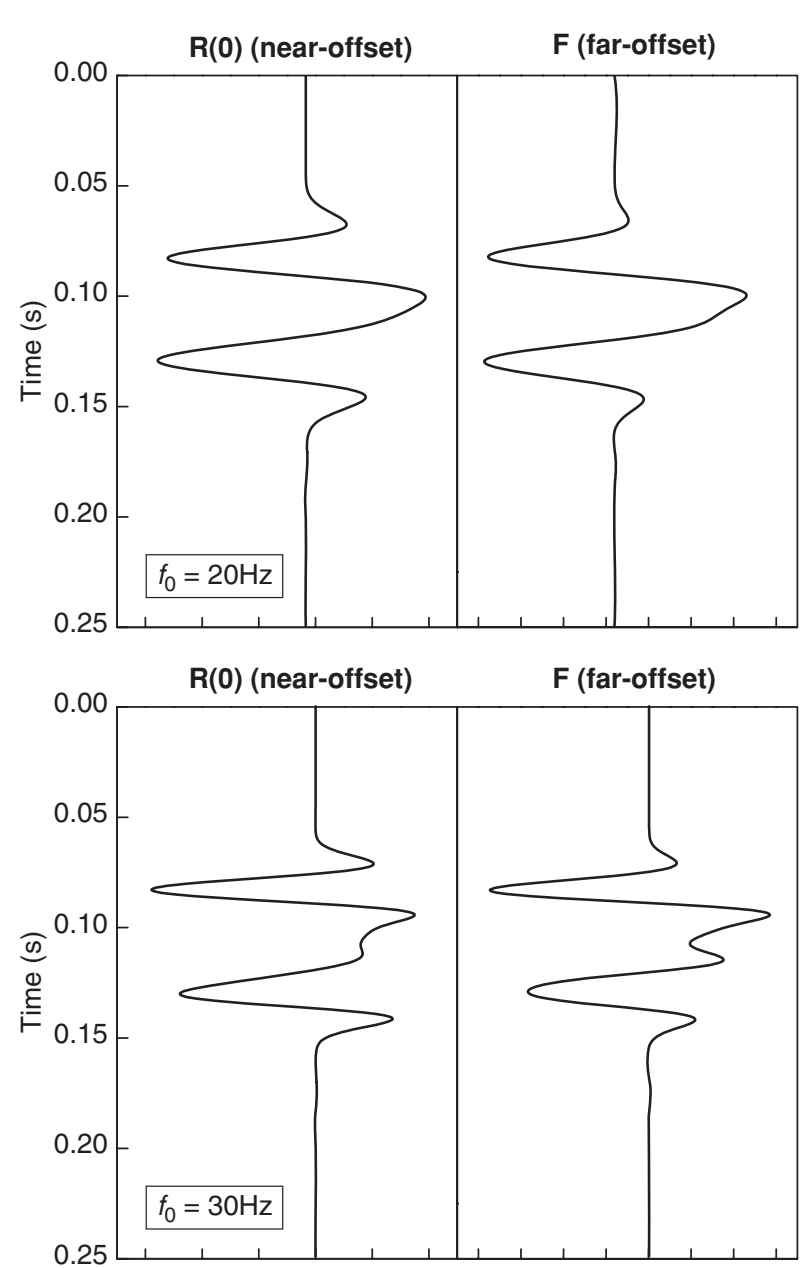

Figure 7

The near and far-offset attributes computed with full wave modelling from the models shown in Figure 6. The central frequency of the wavelet is $20 \mathrm{~Hz}$ (top) and $30 \mathrm{~Hz}$ (bottom).

Fourier spectrum of a given wavelet (in numerical examples we use the Ricker wavelet) and performing the inverse Fourier transform results in AVO attributes as the function of time. In practical seismic applications it is also common to use the near- and far-offset stacks instead of the AVO attribute sections. To compute the far-offset stack one can use Equation (10) for given incident angle $\theta$ with typical $\theta=30^{\circ}$.

\section{NUMERICAL EXAMPLE}

To test our method we use the porosity and net-to-gross synthetic logs computed in SBED model with sedimentation conditions based on the turbidite system from the Glitne Field. In Figure 3 we show these plots for $80 \mathrm{~m}$ thickness of reservoir. First we consider the homogeneous fluid saturation in reservoir. The P-wave velocity, S-wave velocity and density log for fully oil and water saturation cases are shown in Figure 4. The anisotropy parameters logs are also shown in Figure 4. One can see that the water saturation results in increase in both anisotropy parameters, but parameter remains negative. The synthetic near- and far-offset seismic traces computed for both fluid saturation scenarios for seismic wavelet with central frequency of 20 and $30 \mathrm{~Hz}$ are shown in Figure 5. Water saturation results in amplitude increase in the mid-reservoir section for both central frequencies. The oil-water contact (OWC) scenario (20\% water saturation above and $90 \%$ water saturation below the OWC) results in elastic properties shown in Figure 6. The position for OWC is quite pronounced in elastic properties. The synthetic near- and far-offset traces (Fig. 7) results in more smooth reflection in the mid-reservoir section.

\section{CONCLUSIONS}

We propose new method to compute synthetic AVO attributes from ultra-thin reservoirs based on the phantom porosity and net-to-gross logs from SBED model. We simulate also different fluid saturation scenario including the oil-water contact case.

The advantages of proposed technology are following:

- the sedimentology scenario;

- the fluid saturation scenario;

- the AVO attributes from ultra-thin layered reservoirs taking into account the interbedded multiples.

\section{ACKNOWLEDGEMENTS}

We would like to acknowledge the Subseismic project for financial support.

\section{REFERENCE LIST}

Avseth, P. Mukerji, T. and Mavko G. (2005) Quantitative Seismic Interpretation, Cambridge University Press.

Backus, G.E. (1962) Long-wave elastic anisotropy produced by horizontal layering. J. Geophys. Res., 67, 4427-4440.

Gassmann, F. (1951) Uber die Elastitat poroser Medien. Vier. Der Natur. Gesellschaft in Zurich, 96, 1-23.

Kennett, B.L.N. (1983) Seismic wave propagation in stratified media, Cambridge University Press.

Nordahl, K., Ringrose, P.S. and Wen, R. (2005) Petrophysical characterization of a heterolithic tidal reservoir interval using a processbased modeling tool. Petrol. Geosci., 11, 17-28.

Shapiro, S.A. and Hubral, P. (1999) Elastic waves in random media. Fundamentals of seismic stratigraphic filtering, Springer-Verlag.

Stovas and Ursin (2005) The reflection and transmission responses of a periodic layer medium, SEG Annual Meeting, Houston, Extended Abstracts. 
Stovas, A. and Arntsen, B. (2006) Vertical propagation of lowfrequency waves in finely layered media. Geophysics, 71, T87-T94.

Stovas, A., Landrø, M., and Avseth, P. (2006) AVO attribute inversion for finely layered reservoirs. Geophysics, 71, C25-C36.
Wen, R. (2004) 3D modeling of stratigraphic heterogeneity in channelized reservoirs: methods and applications in seismic attribute facies classification. CSEG Recorder, 29, 38-45.

Final manuscript received in December 2006 